\title{
3D MODELLING OF DUNE ECOSYSTEMS USING PHOTOGRAMMETRY FROM REMOTELY PILOTED AIR SYSTEMS SURVEYS
}

\author{
JOSE IGNACIO PAGÁN, ISABEL LÓPEZ, LUIS BAÑÓN \& LUIS ARAGONÉS \\ Department of Civil Engineering, University of Alicante, Spain
}

\begin{abstract}
Dune ecosystems play a key role in coastal dynamics, where a constant exchange of sediments between the sand dune and the beach exists. Thus, it is essential to measure with high precision its movements and monitor its evolution. Classical topographical surveys - even those based on real-time kinematic GPS surveys - are suitable for obtaining profiles with high accuracy, but their cost, in terms of time and personnel, make them inappropriate for large areas. Moreover, these techniques do not precisely measure volumetric changes in high-variable environments such as sand dunes. LiDAR is an active remote sensing method that reflects laser pulses off the land and records topographic positions, but LiDAR scanners are still an expensive and evolving technology. The use of Remotely Piloted Air Systems (RPAS) aims to solve this problem. RPAS map sand dunes using photogrammetric methods, such as Structure-from-Motion (SfM), to produce three-dimensional models from two-dimensional images. This methodology has the advantage of needing only a digital camera and is, therefore, cost effective. The objective of this study is to demonstrate the use of RPAS to map and model the sand dunes of Guardamar del Segura (Spain). The survey covered an extension of 226,000 $\mathrm{m}^{2}$. The results allow us to obtain a point cloud with a density of 30 points per sq $\mathrm{m}$. A 3D digital elevation model of the dune has been created from the dense point cloud, with a vertical accuracy of $0.2 \mathrm{~m}$ Root Mean Square Error. An orthophoto was also created with a spatial resolution of $5 \mathrm{~cm}$. The main conclusion is that the use of RPAS and SfM is an adequate technique to periodically monitor dune ecosystems with high accuracy and cost savings.

Keywords: RPAS, dune, photogrammetry, DSM, SfM, cloud point.
\end{abstract}

\section{INTRODUCTION}

The accurate knowledge of the dune-beach ecosystem is essential, since it is a dynamic area where the interaction between terrestrial and marine ecosystems takes place. Moreover, in these areas there is a constant exchange of sediments with the sea. These natural interactions can be altered by human action, modifying coastal dynamics, causing alterations in beaches and dunes, and affecting their stability [1].

Coastal dunes are difficult landforms to study due to the complex interaction between topography, vegetation and the aeolian processes that move the sand throughout the system. To monitor the changes, it is necessary to repeatedly measure the topography over time. Collecting precise data using classical topographical surveys is both labour-intensive and time-consuming, even using modern techniques such as real time kinematic GPS surveys. Furthermore, volumetric measurements become difficult to obtain due to the low resolution of the data. Light detection and ranging (LiDAR) systems deployed on low-level aircraft, have proven to give accurate horizontal and vertical measurements [2]. However, their cost usually relies on government-sponsored flights, affecting the temporal coverage of the available data.

In recent years, the emergence of affordable unmanned aerial vehicles (RPAS, acronym for Remotely Piloted Aircraft System) for civilian use, together with the development of computational techniques based on images, such as Structure from Motion (SfM), has dramatically increased the use of photogrammetry based on RPAS to produce high-resolution 
digital surface models (DSM) for the study of different surface processes [3]. SfM uses several digital images and automatically solves the geometry of the scene and camera position and orientation by means of an iterative bundle adjustment procedure [4]. SfM, along with multi-view stereo (MVS) algorithms, enables the generation of dense 3D point clouds from a set of digital photos. The use of Ground Control Points (GCPs) with known coordinates enables the projection of the photogrammetric results to a system of absolute coordinates [5]. SfM can be applied to the geomorphological analysis of landforms and their evolution by means of 3D models [6].

Once the data have been obtained, it is necessary to manipulate them in order to analyse spatial characteristics, detect changes and to represent them cartographically. Geographic Information System (GIS) technology allows such analysis [7]. RPAS-based photogrammetry has several advantages in comparison with the methods mentioned before. It can replace the conventional surveys carried out in current coastal monitoring programs, with a considerable reduction in data acquisition costs while maintaining the quality aerial imagery data and increasing the topographic accuracy [6]-[7].

This study is focused on the beaches of Guardamar del Segura, Alicante (Spain), where the continued regression of the shoreline [8] has led to the destruction of buildings located within the Maritime and Terrestrial Public Domain (DPMT) and eroded the dune system. A campaign of flights with a RPAS has been conducted to map and model the sand dunes and the beach. The main aim of this work is to illustrate the procedure of field data collection and the SfM-based photogrammetric modelling performance.

\section{FIELD CAMPAIGN AND DATA ACQUISITION}

The field campaign and data acquisition occurred on June 2017. The area of study encompasses the coastal area that extends from the north of the town of Guardamar del Segura (Alicante, Spain) to the Segura River mouth. The area of interest is the stretch between the shoreline and the inland road, covering an extension of $226,000 \mathrm{~m}^{2}$. It forms a rectangle with a length of $1,500 \mathrm{~m}$ and an width of $150 \mathrm{~m}$ (Fig. 1).

\subsection{Image acquisition}

The data used in this study were obtained using a DJI Phantom 4 Advanced quadcopter. The RPAS is equipped with a 1" CMOS sensor camera (20 Mpixel of resolution). Its focal length ranges from $8.8 \mathrm{~mm}$ to $24 \mathrm{~mm}$. The $5870 \mathrm{mAh}$ battery allows a flight time of aproximately 30 minutes, so the flight planning was developed to cover in 3 passes the entire study area at $60 \mathrm{~m}$ above sea level. The latitudinal overlap was $60 \%$ and the longitudinal overlap was $75 \%$. The spatial resolution of the images at this altitude is $2 \mathrm{~cm}$ per pixel. 1,200 images were recorded. The meteorological conditions were fair, the wind speed was weak and regular, so the flights occurred without any obstacles.

\subsection{GNSS survey}

GCPs are required for the georeferencing. 150 GCPs were acquired using a Leica Viva GS16 GPS reciever, eqquiped with Real Time Kinematic (RTK) technology used to enhance the precision of position data derived from the GNSS (Fig. 1). A topographic GNSS measurements with 2069 points was also surveyed to compare and contrast the data acquired by RPAS. In order to evaluate vertical and horizontal errors, a total of 531 complementary GNSS measurements were carried out on stable areas of the study site (road and wooden walkways). 

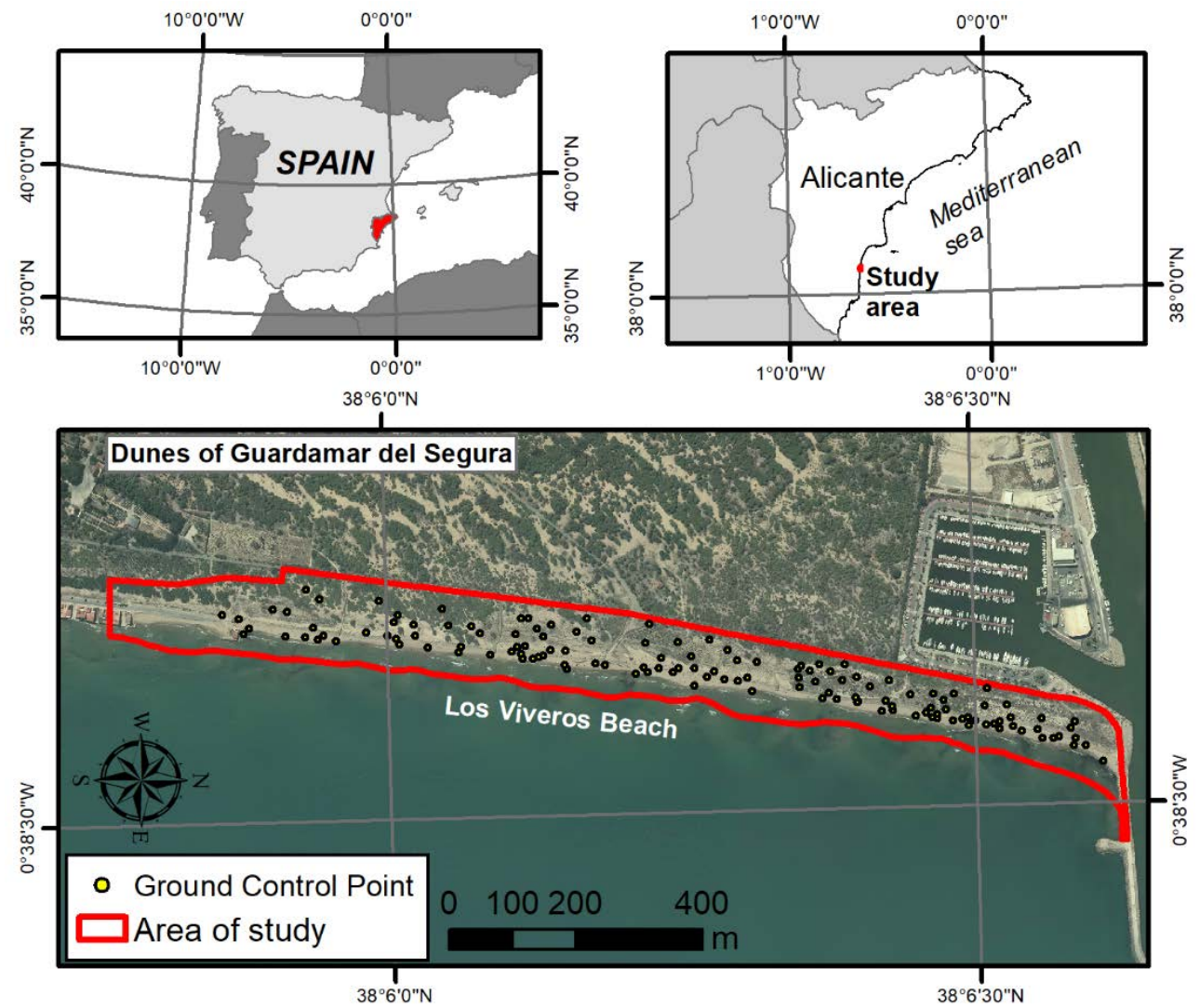

Figure 1: Location of the study area.

\section{SFM-BASED PHOTOGRAMMETRIC PROCESS}

The photogrammetric process based on SfM algorithm is required for 3D coastal topography reconstruction from 2D RPAS images. The SfM algorithm allows reconstruction of a 3D scene geometry by solving the geometry of the scene, camera positions and orientation, extracted from a set of multiple overlapping images [4]. Recently, several photogrammetric software packages based on the SfM method that bring knowledge from computer vision and traditional photogrammetry together, have been developed and are more dedicated to the processing of RPAS images. One commercial package, Agisoft Photoscan [9], was chosen for this study due to its essentially automatic process, based on multi-view 3D reconstruction technology, and its suitability for RPAS image processing. The general workflow for the RPAS images processing is divided into 8 different steps (Fig. 2).

The first step consists of loading the photos from the RPAS survey and its camera positions, saved in the EXIF metadata of the images. This information contains the coordinates of the GNSS signal received by the RPAS and its orientation. In the second stage, Align photos, PhotoScan finds matching points between overlapping images, estimates the camera position for each photo and builds the sparse point cloud model. 


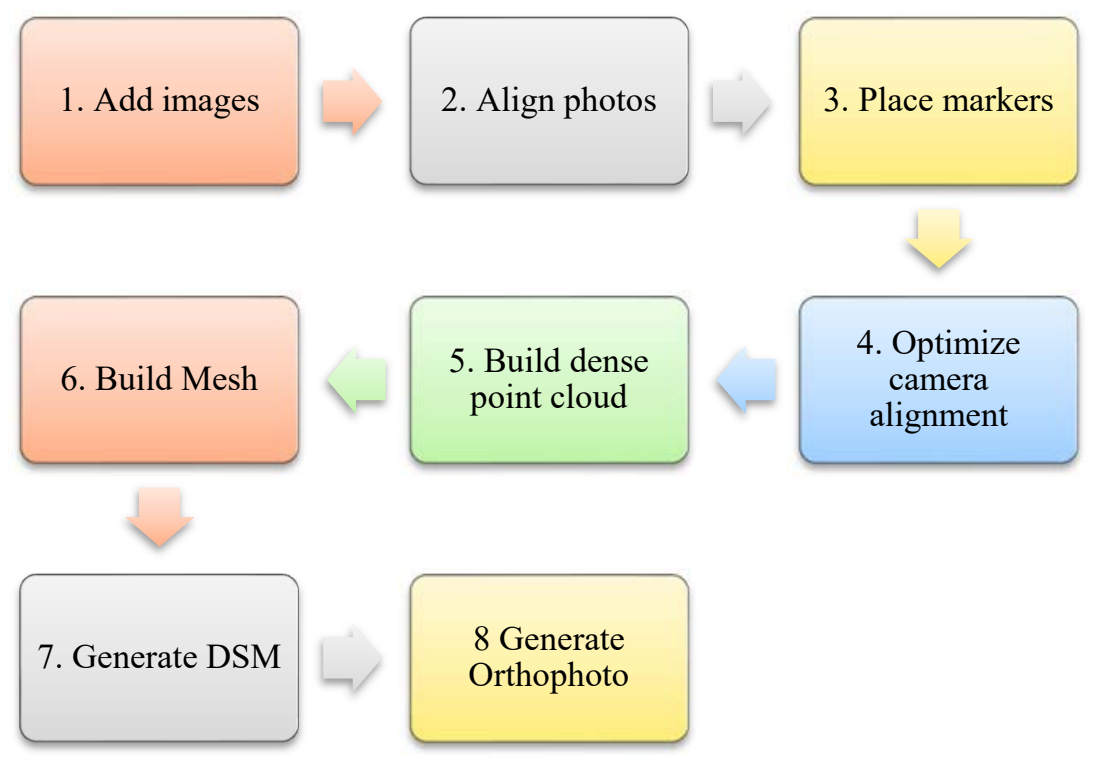

Figure 2: Worflow followed for SfM modelling.

The third stage is placing markers. Markers are used to optimize camera positions and orientation data, which allows for better model reconstruction results. This stage consists of locating the GCPs visible on each photo and place a marker in the corresponding point of the image. The software automatically identifies the position of the markers on every related photo but checking the marker location and refining its position is necessary to provide maximum accuracy. This step is the most time-consuming. Finally, marker coordinates are imported from a file containing the accurate coordinates of the GCPs from the GNSS survey.

The next step is to optimize camera alignment. The position of the images is recalculated considering the established GCPs, so that the result will already be perfectly georeferenced in its XYZ coordinates (Fig. 3).

In stage five the software calculates the depth information for each camera to be combined into a single dense point cloud. After the dense point cloud has been reconstructed it is possible to generate a polygonal mesh model based on the dense cloud data (Fig. 4). Finally, the result output can be exported as a raster DSM and as an orthophoto with a spatial resolution of $5 \mathrm{~cm} /$ pixel, both in the desired coordinate system in a GeoTIFF format.

The SfM technique demands high computing capability for processing and viewing. Thus, the area of study was divided into 3 zones coinciding with the 3 flights performed. These zones were processed independently with the aim of not overloading the capacity of processing of the computer used, which had the following characteristics:

- Windows 10 64-bit.

- Intel Core i7-2670QM @ 2.2 GHz 4 cores 8 threads 6 MB cache.

- 8 Gb RAM.

- 250 GB SSD disk Samsung 850 EVO. 


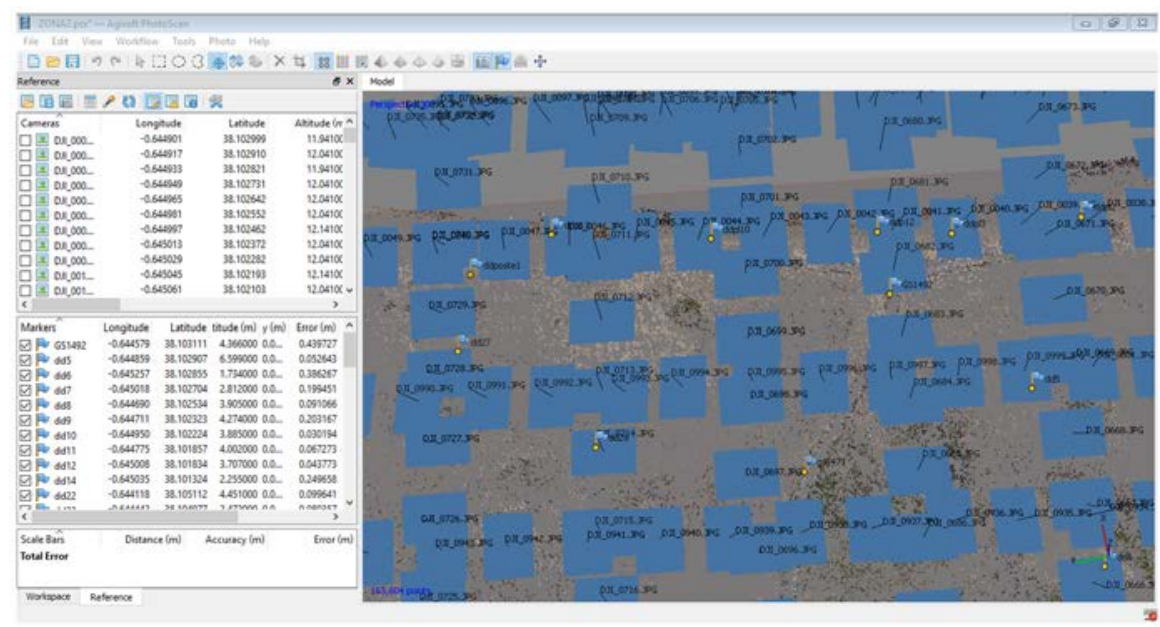

Figure 3: Snapshot with the position of the images and the markers of the GCP identified on the photos. The panel on the left shows the coordinates of the images and the GCPs as well as the errors of the georeferencing process.

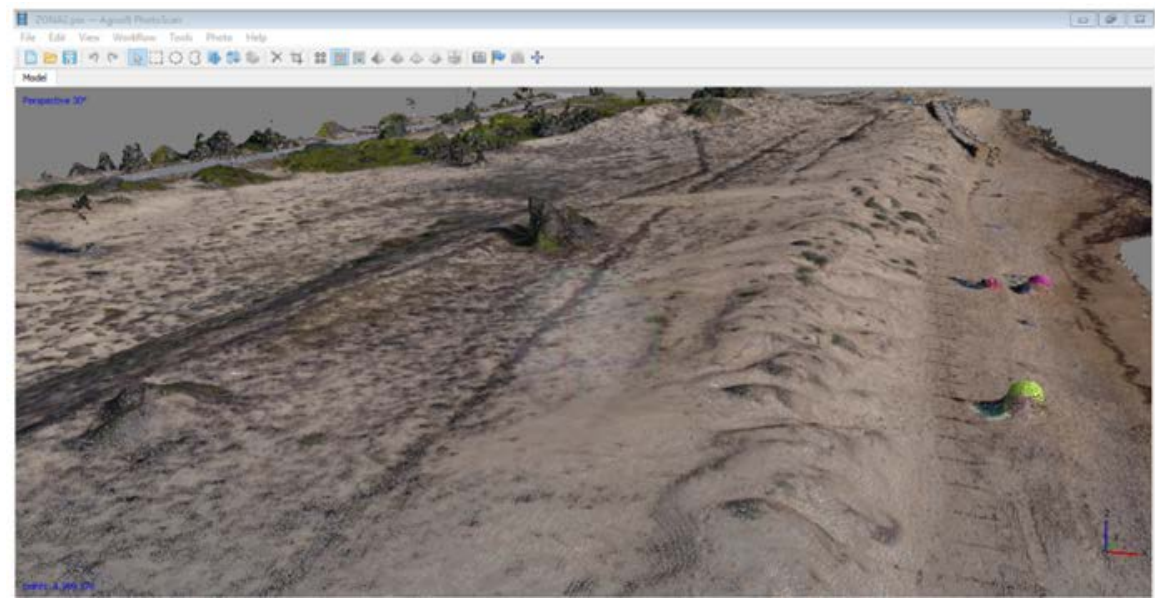

Figure 4: 3D model view of the dune-beach with the dense cloud point coloured in RGB.

\section{RESULTS}

The dune-beach system of Guardamar del Segura has been revealed as an excellent test site for the methodology proposed in this research. The complex dune ecosystem and the adjacent beach have been surveyed using a small, commercial RPAS. Although the area of interest extends over $226,000 \mathrm{~m}^{2}$, the actual survey covered $286,100 \mathrm{~m}^{2}$.

The performance of each stage of the SfM process is shown in Table 1. The most timeconsuming step is placing markers, since it requires the human visual identification of each GCP on all the images. Due to the high number of images and GCP surveyed (8-12 GCP visible per image), to complete this step an average of $5 \mathrm{~h}$ was required. Besides that, aligning 
photos is the second time-consuming step, followed by the building of the dense point cloud. Note that these times depend on the computer processing capacity available, so they can be improved using more powerful PCs. The total time spent to complete all the steps of the SfM methodology was $33 \mathrm{~h}$. However, considering the commitment of time and manpower to conducting traditional field surveys, SfM photogrammetry from RPAS has a better performance.

Table 1: Performance of the SfM process.

\begin{tabular}{|l|l|l|l|}
\hline Stage & Zone 1 & Zone 2 & Zone 3 \\
\hline 1 Add photos & $0: 00: 30$ & $0: 00: 26$ & $0: 00: 46$ \\
\hline 2 Align photos & $3: 13: 00$ & $3: 05: 00$ & $3: 08: 00$ \\
\hline 3. Place markers & $4: 45: 00$ & $5: 15: 00$ & $4: 20: 00$ \\
\hline 4. Optimize alignment & $0: 01: 59$ & $0: 01: 14$ & $0: 02: 28$ \\
\hline 5. Build dense point cloud & $2: 27: 00$ & $2: 49: 00$ & $1: 44: 00$ \\
\hline 6. Build mesh & $0: 27: 50$ & $0: 39: 53$ & $0: 22: 02$ \\
\hline 7. Generate DSM & $0: 05: 12$ & $0: 08: 22$ & $0: 04: 48$ \\
\hline 8. Generate orthophoto & $0: 04: 29$ & $0: 06: 22$ & $0: 03: 59$ \\
\hline Total & $11: 05: 00$ & $12: 05: 17$ & $9: 46: 03$ \\
\hline
\end{tabular}

The results from each zone are shown in Table 2. After processing the zones independently, the dense cloud point, DSM (Fig. 5) and orthophoto (Fig. 6) were merged into one single dataset for the whole area of interest. A total of 8,335,766 points were obtained from the dense point cloud generated, modelling the surface with a density of 30 points per sq $\mathrm{m}$. A 3D digital surface model of the dune and the beach was created from the dense point cloud (Fig. 5), with an average horizontal accuracy of $0.08 \mathrm{~m}$ and a vertical accuracy of 0.2 $m$ RMSE Z. The orthophoto generated from the images captured from the RPAS and the DSM had a spatial resolution of $2.25 \mathrm{~cm}$ per pixel, identifying even the footsteps on the sand dune (Fig. 6(d)).

Table 2: Results of the SfM process.

\begin{tabular}{|l|c|c|c|}
\hline Parameter & Zone 1 & Zone 2 & Zone 3 \\
\hline Number of images & 622 & 689 & 548 \\
\hline Flying altitude & $61 \mathrm{~m}$ & $59 \mathrm{~m}$ & $59 \mathrm{~m}$ \\
\hline Coverage area & $98,600 \mathrm{~m}^{2}$ & $79,500 \mathrm{~m}^{2}$ & $108,000 \mathrm{~m}^{2}$ \\
\hline Number of GCP & 62 & 50 & 42 \\
\hline RMSE XY & $0.098 \mathrm{~m}$ & $0.063 \mathrm{~m}$ & $0.069 \mathrm{~m}$ \\
\hline RMSE Z & $0.238 \mathrm{~m}$ & $0.231 \mathrm{~m}$ & $0.229 \mathrm{~m}$ \\
\hline Dense Cloud Points & $2,792,354$ & $2,306,957$ & $3,236,455$ \\
\hline DSM Point density & 29.6 points per m & 32.7 points per m & 31.8 points per m \\
\hline Ground resolution & $2.3 \mathrm{~cm} /$ pix & $2.19 \mathrm{~cm} / \mathrm{pix}$ & $2.21 \mathrm{~cm} / \mathrm{pix}$ \\
\hline
\end{tabular}




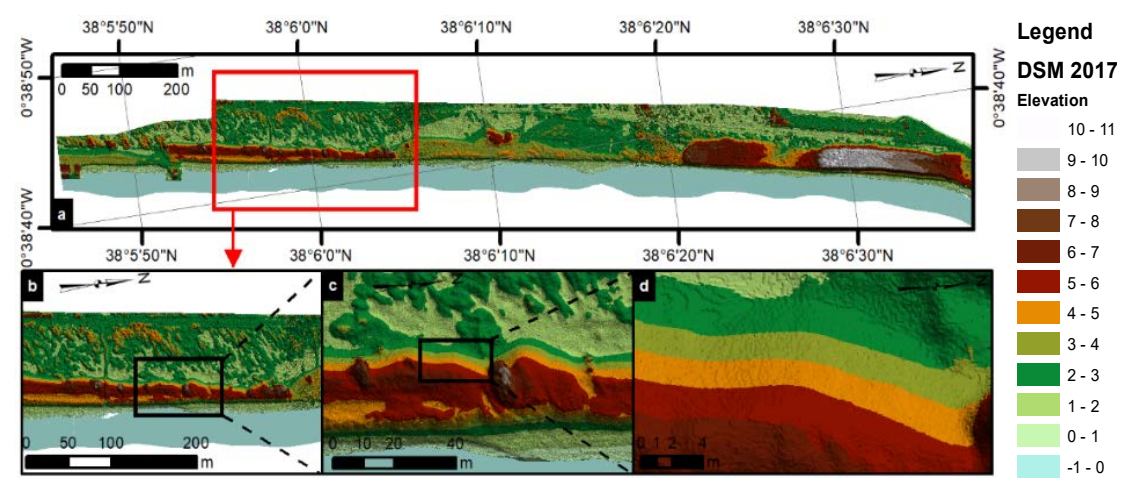

Figure 5: (a) DSM of the area of study; (b) Detail at 1:10000 scale; (c) Detail at 1:2500 scale; and (d) Detail at 1:500 scale.

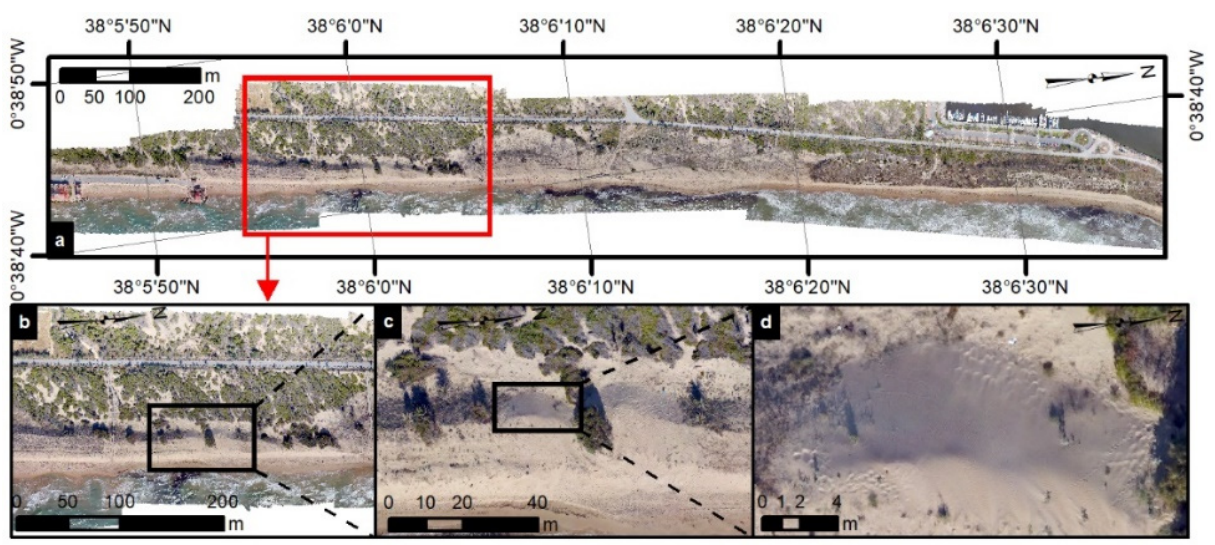

Figure 6: (a) Orthophoto of the area of study; (b) Detail at 1:10000 scale; (c) Detail at 1:2500 scale; and (d) Detail at 1:500 scale.

The dense cloud point can be exported to other software, like Cloud Compare or GIS environments, for better visualization or to compare it with other 3D points clouds available, such as LiDAR datasets. In addition, the DSM and orthophoto can be also exported for the same purpose, incrementing the possibilities of analysis (Fig. 7). For instance, seasonal sediment balance between dune and beach, or analysis of the slopes or dune erosion can be performed.

However, there are some drawbacks to this approach. Firstly, flights regulations can be restrictive on the areas to survey, especially near to populated areas. Secondly, the SfM technique generates a dense point cloud of the surface that can be converted in a Digital Surface Model (DSM), but height can come from the top of buildings, tree canopy, powerlines and other features. However, a bare-earth elevation model is particularly useful in some engineering fields, such as in hydrology, soils and land use analysis. Although there are some techniques to avoid this problem, they usually require the use of Near Infrared (NIR) sensors to better detect and filter vegetation [10], but unfortunately commercial RPAS are not equipped with them. 


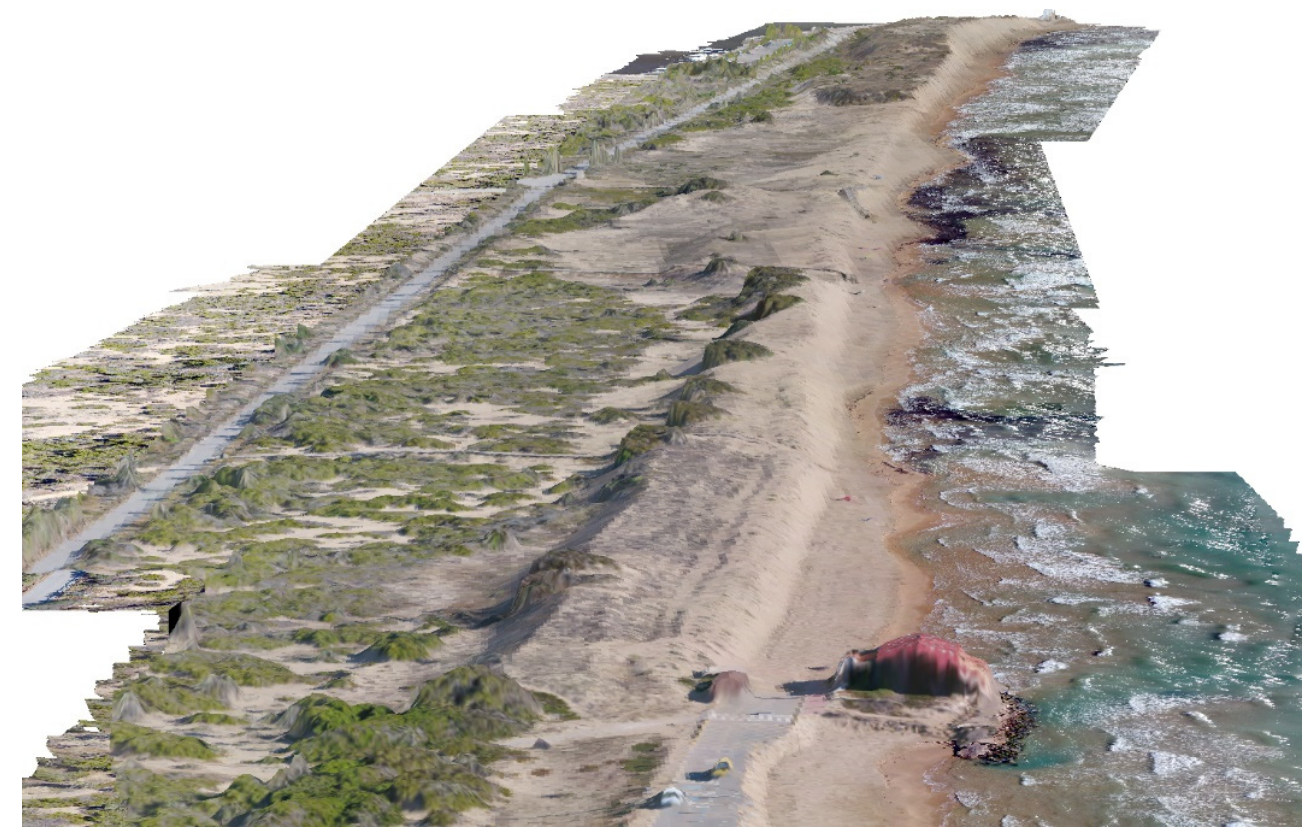

Figure 7: 3D view of the dune-beach ecosystem.

\section{CONCLUSION}

The advantages of RPAS together with SfM methodology are clear: a fast, reliable and highly accurate technique that allows to model complex and changing environments such as dune ecosystems with a scarce inversion. Moreover, analysing the evolution of these systems regularly and in detail are proved as possible in this research.

\section{REFERENCES}

[1] Aragonés, L., Pagán, J.I., López M.P. \& García-Barba, J., The impacts of Segura River (Spain) channelization on the coastal seabed. Science of The Total Environment, 543, pp. 493-504, 2016.

[2] Woolard, J.W. \& Colby, J.D., Spatial characterization, resolution, and volumetric change of coastal dunes using airborne LIDAR: Cape Hatteras, North Carolina. Geomorphology, 48(1-3), pp. 269-287, 2002.

[3] Colomina, I. \& Molina, P., Unmanned aerial systems for photogrammetry and remote sensing: A review. Journal of Photogrammetry Remote Sensing, 92, pp. 79-97, 2014.

[4] Snavely, N., Seitz, S.M. \& Szeliski, R., Modeling the World from Internet Photo Collections. International Journal of Computer Vision, 80(2), pp. 189-210, 2008.

[5] Riquelme, A., Del Soldato, M., Tomás, R., Cano, M., Jordá Bordehore, L. \& Moretti, S., Digital landform reconstruction using old and recent open access digital aerial photos. Geomorphology, 329, pp. 206-223, 2019.

[6] Westoby, M.J., Brasington, J., Glasser, N.F., Hambrey, M.J. \& Reynolds, J.M., "Structure-from-Motion" photogrammetry: A low-cost, effective tool for geoscience applications. Geomorphology, 179, pp. 300-314, 2012.

[7] Andrews, B.D., Gares, P.A. \& Colby, J.D., Techniques for GIS modeling of coastal dunes. Geomorphology, 48(1-3), pp. 289-308, 2002. 
[8] Pagán, J.I., López, I., Aragonés, L. \& García-Barba, J., The effects of the anthropic actions on the sandy beaches of Guardamar del Segura, Spain. Science of The Total Environment, 601-602, pp. 1364-1377, 2017.

[9] Agisoft, Agisoft PhotoScan Professional Edition, 2016.

[10] Kyratzis, A.C., Skarlatos, D.P., Menexes, G.C., Vamvakousis, V.F. \& Katsiotis, A., Assessment of vegetation indices derived by UAV imagery for durum wheat phenotyping under a water limited and heat stressed Mediterranean environment. Plant Science, 8(1114), 2017. 